\title{
Exploring the association of early life physical activity and risk of dementia: a systematic review.
}

Francesco Della Gatta*1-2, Eleonora Lacorte ${ }^{3}$, Elisa Fabrizi ${ }^{1}$, Giulia Remoli ${ }^{3}$, Virginia Cipollini ${ }^{4}$, Fernanda Troili ${ }^{4}$, Nicola Vanacore ${ }^{3}$, Franco Giubilei ${ }^{1}$

${ }^{1}$ NESMOS Department, Faculty of Medicine and Psychology, Sapienza University of Roma, Italy

${ }^{2}$ Escuela de Doctorado Universidad Catolica de Valencia San Vicente Martir, Valencia, Spain

${ }^{3}$ Italian National Institute of Health, Italy

${ }^{4}$ Department of Human Neuroscience, Sapienza University of Roma, Italy

Running title: physical activity and dementia

*Corresponding author:

Francesco Della Gatta

Key words: physical activity, dementia, prevention, risk factors, cognitive impairment 


\begin{abstract}
Introduction

Physical activity has been included in the list of twelve modifiable risk factors for dementia, despite conflicting results from observational and controlled studies. In particular it is not clear whether physical inactivity near the time of dementia diagnosis is a consequence or cause of dementia. We review all available studies reporting the possible association between having engaged in PA before 60 years of age and the risk of dementia.
\end{abstract}

\title{
Evidence acquisition
}

We performed a systematic review based on the methodology reported in the Cochrane handbook for systematic reviews and following the PRISMA statement. Bibliographic searches were carried out on the databases PubMed, ISI Web of Science and the Cochrane Database of Systematic Reviews. Further references were retrieved from published systematic reviews on the same topic. Included studies were assessed using the Newcastle Ottawa scale.

\section{Evidence synthesis}

The bibliographic search yielded 1381 records. A total of 11 studies were included. Three of the included studies were case control studies, while the remaining 8 were cohort studies The overall quality of included studies was high. However, clinical criteria for the diagnosis of dementia, criteria to define and measure and PA and time-reference of exposure were heterogeneous, with some studies considering specific age range of exposure, and other reports dealing with more generic "adult age".

\section{Conclusions}

This review suggests that there is insufficient evidence to conclude whether PA in early life may affect the incidence of dementia in later life. Studies in this field are very complicated and recognizing the impact of PA in early life given all the confounding factors is very difficult. Further studies are warranted. In these studies, it will be crucial to define the type, quantity and intensity of PA as well as to stratify analysis by sex, cultures and social classes. 


\section{Introduction}

Worldwide around 50 million people are affected by dementia, and this number is expected to increase to about 150 million by 2050 (1), due to ageing population. However, in some highincome countries, comparing similar cohorts at different time, the age-specific incidence of dementia appears to decrease, probably due to lifestyle changes $(2,3)$.

It was recently estimated that around $40 \%$ of dementia cases worldwide might be attributable to 12 modifiable risk factors: less education, physical inactivity, hearing impairment, low social contact, hypertension, diabetes, obesity, depression, smoking, alcohol consumption, traumatic brain injury and air pollution (4). Prevention of these risk factors could therefore be the key to prevent or delay the onset of dementia.

In dementia, risk factor studies are complex for many reasons. As the patterns of these risk factors can change with age and morbidity, knowledge of the life course is crucial to study the risk. This is the case of obesity and hypertension which are risk factors in midlife but not in later life $(5$, 6). There are two different mechanisms of protection from dementia: reduce neuropathological damage and increase and maintain "brain reserve". The latter is changeable and difficult to quantify due to the many risk factors that influence it in early life, such as the level of education and cognitive stimulation (7). On the contrary, midlife and old-age risk factors are associated with cerebral neuropathological damage. Finally, all risk factors can change across sex, cultures and social classes. In this context, it is difficult to understand the true protective value of a single risk factor.

Studying physical activity (PA) is even more complicated. Additional factors may affect the results of studies, such as the difficulties to quantify the activity, fluctuations during life and frequent overlap with other risk factors. Nevertheless, PA has been indicated as a modifiable risk factor for dementia $(8,9)$. The results of longitudinal observational studies and randomized clinical trials are however conflicting. These discrepancies are also present within the same study. The Whitehall Study found that PA reduces dementia risk over 10 years, but not 27-year follow-up (10). A metaanalysis of 19 observational studies of middle-aged people describes an increased incidence of dementia in physically inactive people 10 years before diagnosis (11). This increase in dementia risk, however, was found only in people with cardio-metabolic comorbidities but not in other people. In neurodegenerative dementias, the neuropathological processes underlying the development of irreversible brain damage start many years before clinical diagnosis (12) and neuropsychiatric 
symptoms such as depression and anxiety can increase during this prodromal phase $(13,14)$, changing the lifestyle of people. Therefore, it is not clear whether physical inactivity preceding the diagnosis of dementia is a consequence or cause of dementia.

The aim of this review is to systematically gather all available studies reporting the possible association between having engaged in PA before 60 years of age and the risk of dementia assessing their methodological quality.

\section{Evidence acquisition}

We carried out a systematic review based on the methodology reported in the Cochrane handbook for systematic reviews (15) and following the PRISMA statement for reporting systematic reviews and meta-analyses (16). Bibliographic searches were carried out on the databases PubMed, ISI Web of Science and the Cochrane Database of Systematic Reviews with the following keywords: (dementia* OR Alzheimer* OR “cognitive impairment") AND (sport* OR exercise* OR "physical activity" OR "physical activities" OR fitness) AND ("risk factor" OR "risk factors" OR "prevention" OR "preventive") AND (frequency* OR incidence* OR prevalence* OR cohort OR prospective* OR longitudinal* OR case-control*).

Searches were not limited for language, publication year or study design. The bibliographic references of included studies were also searched to identify further relevant studies. The titles and abstracts of retrieved studies were reviewed by 3 independent reviewers and studies that were pertinent with and relevant to the topic of the review were selected. Disagreements were resolved by consensus.

Selected studies were read in full text and were applied the predefined inclusion/exclusion criteria. Any type of observational study considering the exposure to any type of PA before 60 years of age as a potential protective factor for any type of dementia was included. Only studies reporting the possible association between having engaged in PA before 60 years of age and dementia as one of the main objectives, and only studies reporting enough information to allow for data extraction and methodological assessment were included. Studies reporting narrative results, as well as abstracts, conference proceedings, posters, editorials, letters, narrative reviews, RCTs, case-reports or case-series were excluded. We considered systematic reviews and/or meta-analyses separately to review the references and check for consistency of results. 
The methodological quality of included studies was assessed using the Newcastle-Ottawa Scale (NOS) for quality assessment of non-randomized studies (17). The NOS scale comprises 8 items analyzing the appropriateness of 3 domains: the selection of the study sample, the comparability of groups, and the ascertainment of the exposure for case-control studies or of the outcome for cohort studies. A maximum of 9 stars can be assigned to each study. Specifically, studies can be scored a maximum of 4 stars for Selection, 2 stars for Comparability, and 3 stars for either Outcome or Exposure. The presence of heterogeneity and any other potential bias was also investigated. Data extraction was carried out using standardized forms, and potential disagreements were resolved by consensus.

\section{Evidence synthesis}

Bibliographic search yielded 1381 records, and further four studies were gathered from 12 systematic reviews. A total of 1299 records were excluded as they were not relevant nor pertinent to the topic of the review. The remaining 86 studies were applied the predefined inclusion and exclusion criteria. A total of 75 studies were excluded as they were systematic reviews $(n=12)$, editorial $(n=1)$, exposure measured in participants $\geq 60$ or unspecified $(n=55)$, did not observe incidence of dementia $(n=7)$. A total of 11 studies were included in the qualitative analysis and were categorized according to study design and applied the NOS checklist. The flow diagram of the included literature is reported in Figure 1.

Three of the included studies were case control studies $(18,19,20)$, while the remaining 8 were cohort studies $(10,21,22,23,24,25,26,27)$. The summary of the characteristics and results of each included study is reported in Table $1(10,18-27)$.

The overall quality of included studies was high, with all of the case-control studies scoring 8 at the NOS scale, and all of the included cohort studies scoring 9 at the NOS score except for one that scoring 8 (27). All case-control studies enrolled populations that were representative of general population, and all assessed cases with a structured, validated measure, in both cases and controls. Moreover, all of included case-control studies enrolled pairs of twins, thus minimizing the potential for confounders. Similarly, all of cohort studies enrolled participants who were representative of the general population, as they were all part of larger, historical cohort studies. All of studies reported a standardized, multi-step procedure for the identification of cases, and all of them used standardized, validate measures, except for 2 that used a consensus method based on clinical 
information $(23,27)$. Moreover, all of included studies reported data adjusting for at least a set of basic demographic data.

Five of included studies used the DSM criteria, with 2 case-control studies $(19,20)$ and 1 cohort study (25) classifying diagnoses according to the DSM III-R criteria, and 1 case-control (18) and 1 cohort study (26) using the DSM IV criteria. As for ICD criteria, 1 cohort study used the ICD-7 criteria (22) 2 cohort studies used the ICD-10 criteria (10, 24), 1 cohort study used both ICD-9 and ICD-10 criteria (21) and the remaining 2 studies used consensus criteria based on either clinical data (23) or on medical records (27).

All of the included studies used different criteria to define and measure PA and/or physical exercise, with definitions varying to include PA, physical exercise or leisure activities, and defining categories according to frequency, intensity or MET cutoffs. Moreover, the time-reference of exposure was also heterogeneous, with some studies considering specific age range of exposure, and other reports dealing with more generic "adult age".

All of included case-control studies reported ORs along with their confidence intervals as a measure of association. Two studies enrolled pairs of twins from the Swedish Twin Registry. The first of these studies (18) gathered information on the amount of exercise practiced from age 25 to age 50 , categorizing the variable in a 4-point scale from "hardly any activity" [0] to "hard activity" [3], with "hardly any" used as a reference category within the analyses. Results from the study showed a significant protective effect in both the crude and the adjusted models of light, regular and hard PA on the risk of dementia. However, when analyzing the risk of $A D$, the protective effect resulted significant only for light and regular activity. The second of these studies (20) gathered information on the frequency of participation in a set of 11 activities before the age of 40 years. Results from the analyses reported a marginally significant effect in people participating in these activities on the risk of any type of dementia (OR $0.63,95 \% \mathrm{Cl} 0.39-1.00, \mathrm{p} \leq 0.05$ ) and $A D(O R 0.54$, $95 \% \mathrm{Cl} 0.29-1.00, \mathrm{p} \leq 0.05)$. The third included case-control study (19) enrolled pairs of male twins from the Duke Twins Study of Memory in Aging who were also members of the NAS-NRC Registry of World War II veterans. The study recorded the frequency of participation in physical exercise and leisure activities after age 35, categorizing it as 1 (never) to 2 (sometimes) to 5 (every day). Results were reported for the overall sample and according to type of twins (monozygotic and dizygotic), and showed no significant effect of PA on the risk of dementia. 
All of included cohort studies reported HRs and their confidence intervals as a measure of association, except for 1 study that reported the OR of having dementia among physically active groups vs no-PA group (26). One of the two studies with the youngest samples (24) enrolled participants $\geq 20$ years in the HUNT1 study and gathered information on frequency and intensity of PA performed during a typical week to build a "moderate-to-vigorous PA" (MVPA) variable, using "no MVPA" as a reference for the analyses. Results showed no significant association between MVPA and the risk of dementia. The second (22) study included participants aged $\leq 30$ years who were part of the Harvard Alumni Health Study, a prospective cohort study of men who entered Harvard University between 1916 and 1950, and gathered information on the amount and frequency of physical exertion coding it as $\geq 5$ hours of PA per week or $\leq 4$ hours per week. Results showed no significant association between PA and dementia (HR 0.93, 95\% Cl 0.70-1.23, $p=0.59$ ).

Two studies included subjects in their 30s. The first study (10) enrolled participants in the Whitehall II study aged 35-55 years and gathered information on the frequency and length of engagement in mildly energetic, moderately energetic, and vigorous PA. Using as reference categories $<8$ hours/week for total PA (designating the sum of all physical activity) and $<5$ hours/week for mild PA, analyses showed no association between physical activity and both mild and moderate to vigorous intensity activity and risk of dementia over an average 27-year follow-up. The second study (25) a community-based sample of Swedish women aged 38-60 years collected information on leisure-time physical activity (LTPA) categorizing it in 4 levels: "almost inactive", "some physical activity $\geq 4$ hours/week", "regular exercise", "regular training and competitive sports". Results were reported according for obesity status, with the category "non-obese active" as a reference. They showed a significant increase in the risk of dementia for the category "obese inactive" (HR 3.31, 95\% Cls 1.43-7.66). Three studies included subjects in their 40s. The first study (23) enrolled men aged 42-61 years who participated in the Finnish Kuopio Ischaemic Heart Disease (KIHD) study and used the KIHD 12-month LTPA questionnaire to gather information on total leisure and occupational PA, classifying it in tertiles, with the first tertile used as a reference for the analyses. Results showed no significant protective effect of PA on the risk of dementia for both the second and third tertile. The second study (21) included subjects with a mean age of 47.3 years who participated in the Health Surveys for England and the Scottish Health Surveys. PA was classified, according to frequency, as $<5$ weekly sessions of MVPA compared to $\geq 5$ weekly sessions. Results showed no significant protective effect of engaging in $\geq 5$ weekly sessions of MVPA on the risk of dementia ( $p=0.52$ ). The third study (27) enrolled medical graduates from 1948-1964 participants in 
the Johns Hopkins Precursors study and recorded information on the hours per day spent over a typical week engaging in vigorous activities, moderate activities, or sedentary light activities and sleeping. Results were reported for both full sample and stratified per age class. No significant effect of regular exercise on the risk of dementia was found. The last included cohort studies (26) enrolled participants with a mean age of 51 years who were part of the Age Gene/Environment SusceptibilityReykjavik Study and collected information on their participation in any sport or form of exercise during their adult life. Based on the frequency of participation, PA was categorized as (a) no-PA, (b) $\leq 5$ hours of PA/week, (c) $>5$ hours of PA/week. Results were reported as the OR of having dementia among PA groups vs no-PA group and showed a significant lower OR of dementia in those with $\leq 5$ hours of PA/week (OR $0.59,95 \% \mathrm{Cl} 0.40-0.87$ ) but no significant effect of $>5$ hours/week of PA on the risk of dementia (OR $0.74,95 \% \mathrm{Cl} 0.34-1.62$ ).

\section{Discussion}

Physical activity has been identified as potentially modifiable risk factors for dementia and cognitive decline (4). Furthermore, the estimated population risk for dementia associated with physical inactivity is probably greater than for other risk factors (28).

Based on evidence from both observational and experimental studies, the Alzheimer's Association concludes that PA is associated with a decreased risk of cognitive impairment or with an improved of cognitive function (29). However, the report underlines that evidence is still unclear on the optimal length, type and intensity of the PA, and in what period of a person's lifespan it should be carried out as to maximize its potential protective effect. The difficulty in assessing these aspects might be due to several issues related to the association between PA and cognitive impairment or dementia.

One issue is the role of PA in the causal relationship with cognitive impairment. Observational studies agree that PA is associated with lower short-term risk of dementia and higher cognitive performance in older age $(30,31)$. Furthermore, it has been reported that PA can reduce the progression from Mild Cognitive Impairment to dementia (32). However, these positive findings could also be explained hypothesizing that in ageing people, physical inactivity is a consequence of subclinical cognitive deterioration or of other pathologies associated with ageing such as cardiovascular disease (CVD). It is well-known that PA is an established protective factor for CVD and cardio-metabolic diseases (CMD), which are also risk factors for dementia. People with a higher risk of CVD could be led to engage in PA at a certain point of their life, but some other could also 
stop to engage in PA due to the onset of CVD or CMD. Moreover, some people might stop to participate in PA due to the onset of the initial symptoms of cognitive decline such as behavioral disorders. Therefore, measuring PA in a time span to close to the onset of the disease could be a confounding factor.

Another point is the real influence that the risk factors have on cognition at a time of higher biological vulnerability of the brain such as the time near the clinical onset of dementia. The neuropathological changes underlying dementia put the brain in a "critical state" in which the risk factors might have more weight. Trying to reduce the impact of all these issues, we planned this review by only including studies that evaluated exposure to PA in early life and middle age.

Although the overall quality of the included studies is high, the results of this review are conflicting. Only three studies $(18,20,26)$ reported a significant effect of having engaged PA starting before the age of 60 on the later risk of dementia. This could be due to a heterogeneity in the measures of PA and to the difficulty of retrieving data on PA in very long cohorts. All the included studies used different criteria to define and measure PA and/or physical exercise. Most of the included studies measured the PA in a reductive way analyzing only physical exercises and/or some leisure activities. Notably, the study that took into account the greatest number of physical, leisure and social activities showed a reduction on the later risk of dementia (20). At this regard, we must keep in mind that many risk factors overlap and PA is strongly linked to education in early life (33). Finally, the time-reference of exposure was also heterogeneous, with studies considering an age of exposure either in a specific age range or more generally in "adult age". This also prevents direct comparison and combining of evidence from different studies due to these large differences in the measurement of the exposures (34).

To increase consistency of this review, we only included studies that had the diagnosis of dementia as primary endpoint. We found that several clinical criteria were used for the diagnosis, such as ICD and DSM criteria. This can significantly affect the number of subjects with dementia in the included studies. The percentage of subjects with dementia rages from 3.1 percent with ICD-10 criteria to 29.1 percent when the DSM-III criteria are used (35). This is a further limitation when comparing the results of the studies.

Two studies suggested an association between early-life PA and cognitive functioning later in life $(36,37)$. These studies were not included in this review because did not consider the diagnosis of dementia as an endpoint. One study reported that women who were physically inactive at various 
points in their life, in particular at teenage, had a higher risk of cognitive impairment and poorer cognitive performance in old age (36). Another study reported a positive association in men between regular PA early in life and improved cognitive performance, in particular information processing speed, at older age (37). These results support the hypothesis that people engaging in PA from a younger age should have better cognitive performance later in life. Different mechanisms can justify this hypothesis: upregulated hippocampal levels of brain-derived neurotrophic factor, improved cerebral blood flow, decreased secretion of brain-toxic stress hormones such as cortisol, reduced CVD and cerebrovascular disease, reduced other risk factors such as hypertension and obesity $(38,39,40)$.

In conclusion, although PA can affect positively the cognitive performance in every moment of life, this review suggests that there is insufficient evidence to conclude whether PA in early life may affect the incidence of dementia in later life. Studies in this field are very complicated and recognizing the impact of $\mathrm{PA}$ in early life given all the confounding factors is very difficult. Longitudinal investigations of the effect of early life PA on dementia risk in elderly people have many limitations and produce inconsistent results. Therefore, further studies are warranted. In these studies, it is crucial to define the type, quantity and intensity of PA as well as to stratify data analysis by sex, culture and social class. Physical activity should be assessed using objective measurements and differentiating PA at various age should be recommended.

From the public health perspective, however, while waiting for a disease-modifying agent for dementia, non-pharmacological interventions, such as physical exercise, remain the only feasible approach to preventing dementia and can have a considerable effect when taken together to other health and wellbeing benefits. Physical and recreational activity is crucial to improve cognitive performance and have very low costs, which can turn into a great benefit for public health and wellbeing benefits for individuals.

\section{Author Contribution}

All authors of the present systematic review contributed to the concept and drafting of this manuscript. All authors read and approved the final version of the manuscript.

\section{Data Sharing}

The data that support the findings of this study are available from the corresponding author upon reasonable request. 


\section{Conflicts of interest}

The authors certify that there is no conflict of interest with any financial organization regarding the material discussed in the manuscript.

\section{Funding}

This study did not receive specific funding.

\section{References}

(1) Patterson C. World Alzheimer Report 2018. Alzheimer's Disease International (ADI), London. September 2018.

(2) Wu YT, Beiser AS, Breteler MMB, Fratiglioni L, Helmer C, Hendrie HC, et al. The changing prevalence and incidence of dementia over time - current evidence. Nat Rev Neurol. 2017 Jun;13(6):327-339.

(3) Kingston A, Comas-Herrera A, Jagger C; MODEM project. Forecasting the care needs of the older population in England over the next 20 years: estimates from the Population Ageing and Care Simulation (PACSim) modelling study. Lancet Public Health. 2018 Sep;3(9):e447-e455.

(4) Livingston G, Huntley J, Sommerlad A, Ames D, Ballard C, Banerjee S, et al. Dementia prevention, intervention, and care: 2020 report of the Lancet Commission. Lancet. 2020 Aug 8;396(10248):413-446.

(5) Singh-Manoux A, Dugravot A, Shipley M, Brunner EJ, Elbaz A, Sabia S, et al. Obesity trajectories and risk of dementia: 28 years of follow-up in the Whitehall II Study. Alzheimers Dement. 2018 Feb;14(2):178-186.

(6) McGrath ER, Beiser AS, DeCarli C, Plourde KL, Vasan RS, Greenberg SM, et al. Blood pressure from mid- to late life and risk of incident dementia. Neurology. 2017 Dec 12;89(24):2447-2454.

(7) Roe CM, Xiong C, Miller JP, Morris JC. Education and Alzheimer disease without dementia: support for the cognitive reserve hypothesis. Neurology. 2007 Jan 16;68(3):223-8.

(8) Livingston G, Sommerlad A, Orgeta V, Costafreda SG, Huntley J, Ames D, et al. Dementia prevention, intervention, and care. Lancet. 2017 Dec 16;390(10113):2673-2734.

(9) Hersi M, Irvine B, Gupta P, Gomes J, Birkett N, Krewski D. Risk factors associated with the onset and progression of Alzheimer's disease: A systematic review of the evidence. Neurotoxicology. 2017 Jul;61:143-187.

(10) Sabia S, Dugravot A, Dartigues JF, Abell J, Elbaz A, Kivimäki M, et al. Physical activity, cognitive decline, and risk of dementia: 28 year follow-up of Whitehall II cohort study. BMJ. 2017 Jun 22;357:j2709.

(11) Kivimäki M, Singh-Manoux A, Pentti J, Sabia S, Nyberg ST, Alfredsson L, et al. Physical inactivity, cardiometabolic disease, and risk of dementia: an individual-participant metaanalysis. BMJ. 2019 Apr 17;365:I1495.

(12) Gordon BA, Blazey TM, Su Y, Hari-Raj A, Dincer A, Flores S, et al. Spatial patterns of neuroimaging biomarker change in individuals from families with autosomal dominant Alzheimer's disease: a longitudinal study. Lancet Neurol. 2018 Mar;17(3):241-250. 
(13) Wetherell JL, Gatz M, Johansson B, Pedersen NL. History of depression and other psychiatric illness as risk factors for Alzheimer disease in a twin sample. Alzheimer Dis Assoc Disord. 1999 Jan;13(1):47-52.

(14) Ma L. Depression, Anxiety, and Apathy in Mild Cognitive Impairment: Current Perspectives. Front Aging Neurosci. 2020 Jan 30;12:9.

(15) Higgins JPT, Green S (editors). Cochrane Handbook for Systematic Reviews of Interventions Version 5.1.0 [updated March 2011]. The Cochrane Collaboration, 2011. Available from www.handbook.cochrane.org.

(16) Liberati A, Altman DG, Tetzlaff J, Mulrow C, Gøtzsche PC, loannidis JP, et al. The PRISMA statement for reporting systematic reviews and meta-analyses of studies that evaluate healthcare interventions: explanation and elaboration. BMJ. 2009 Jul 21;339:b2700.

(17) Wells GA, Shea B, O'Connell D, Peterson J, Welch V, Losos M, et al. The Newcastle-Ottawa Scale (NOS) for Assessing the Quality of Nonrandomized Studies in Meta-Analyses. 2011. Available online at: http://www.ohri.ca/programs/clinical_epidemiology/oxford.asp.

(18) Andel R, Crowe M, Pedersen NL, Fratiglioni L, Johansson B, Gatz M. Physical exercise at midlife and risk of dementia three decades later: a population-based study of Swedish twins. J Gerontol A Biol Sci Med Sci. 2008 Jan;63(1):62-6.

(19) Carlson MC, Helms MJ, Steffens DC, Burke JR, Potter GG, Plassman BL. Midlife activity predicts risk of dementia in older male twin pairs. Alzheimers Dement. 2008 Sep;4(5):324-31.

(20) Crowe M, Andel R, Pedersen NL, Johansson B, Gatz M. Does participation in leisure activities lead to reduced risk of Alzheimer's disease? A prospective study of Swedish twins. J Gerontol B Psychol Sci Soc Sci. 2003 Sep;58(5):P249-55.

(21) Batty GD, Russ TC, Starr JM, Stamatakis E, Kivimäki M. Modifiable cardiovascular disease risk factors as predictors of dementia death: pooling of ten general population-based cohort studies. J Negat Results Biomed. 2014 May 23;13:8.

(22) Russ TC, Lee IM, Sesso HD, Muniz-Terrera G, Batty GD. Five-decade trajectories in body mass index in relation to dementia death: follow-up of 33,083 male Harvard University alumni. Int J Obes (Lond). 2019 Sep;43(9):1822-1829.

(23) Kunutsor SK, Laukkanen JA, Kauhanen J, Willeit P. Physical activity may not be associated with long-term risk of dementia and Alzheimer's disease. Eur J Clin Invest. 2021 Mar;51(3):e13415.

(24) Zotcheva E, Bergh S, Selbæk G, Krokstad S, Håberg AK, Strand BH, et al. Midlife Physical Activity, Psychological Distress, and Dementia Risk: The HUNT Study. J Alzheimers Dis. 2018;66(2):825-833.

(25) Mehlig K, Skoog I, Waern M, Miao Jonasson J, Lapidus L, Björkelund C, et al. Physical activity, weight status, diabetes and dementia: a 34-year follow-up of the population study of women in Gothenburg. Neuroepidemiology. 2014;42(4):252-9.

(26) Chang M, Jonsson PV, Snaedal J, Bjornsson S, Saczynski JS, Aspelund T, et al. The effect of midlife physical activity on cognitive function among older adults: AGES--Reykjavik Study. J Gerontol A Biol Sci Med Sci. 2010 Dec;65(12):1369-74.

(27) Gross AL, Lu H, Meoni L, Gallo JJ, Schrack JA, Sharrett AR. Physical Activity in Midlife is not Associated with Cognitive Health in Later Life Among Cognitively Normal Older Adults. J Alzheimers Dis. 2017;59(4):1349-1358.

(28) Norton S, Matthews FE, Barnes DE, Yaffe K, Brayne C. Potential for primary prevention of Alzheimer's disease: an analysis of population-based data. Lancet Neurol. 2014 Aug;13(8):78894. 
(29) Baumgart M, Snyder HM, Carrillo MC, Fazio S, Kim H, Johns H. Summary of the evidence on modifiable risk factors for cognitive decline and dementia: A population-based perspective. Alzheimers Dement. 2015 Jun;11(6):718-26.

(30) Buchman AS, Boyle PA, Yu L, Shah RC, Wilson RS, Bennett DA. Total daily physical activity and the risk of $A D$ and cognitive decline in older adults. Neurology. 2012 Apr 24;78(17):1323-9.

(31) Hollamby A, Davelaar EJ, Cadar D. Increased Physical Fitness Is Associated with Higher Executive Functioning in People with Dementia. Front Public Health. 2017 Dec 21;5:346.

(32) Gill DP, Gregory MA, Zou G, Liu-Ambrose T, Shigematsu R, Hachinski V, et al. The Healthy Mind, Healthy Mobility Trial: A Novel Exercise Program for Older Adults. Med Sci Sports Exerc. 2016 Feb;48(2):297-306.

(33) Phillips C. Lifestyle Modulators of Neuroplasticity: How Physical Activity, Mental Engagement, and Diet Promote Cognitive Health during Aging. Neural Plast. 2017;2017:3589271.

(34) Dacks PA, Andrieu S, Blacker D, Carman AJ, Green AM, Grodstein F, et al. Dementia Prevention: optimizing the use of observational data for personal, clinical, and public health decision-making. J Prev Alzheimers Dis. 2014 Feb;1(2):117-123.

(35) Erkinjuntti T, Ostbye T, Steenhuis R, Hachinski V. The effect of different diagnostic criteria on the prevalence of dementia. N Engl J Med. 1997 Dec 4;337(23):1667-74.

(36) Middleton LE, Barnes DE, Lui LY, Yaffe K. Physical activity over the life course and its association with cognitive performance and impairment in old age. J Am Geriatr Soc. 2010 Jul;58(7):1322-6.

(37) Dik M, Deeg DJ, Visser M, Jonker C. Early life physical activity and cognition at old age. J Clin Exp Neuropsychol. 2003 Aug;25(5):643-53.

(38) Cotman CW, Berchtold NC. Exercise: a behavioral intervention to enhance brain health and plasticity. Trends Neurosci. 2002 Jun;25(6):295-301.

(39) Santos RF, Galduróz JC, Barbieri A, Castiglioni ML, Ytaya LY, Bueno OF. Cognitive performance, SPECT, and blood viscosity in elderly non-demented people using Ginkgo biloba. Pharmacopsychiatry. 2003 Jul;36(4):127-33.

(40) Churchill JD, Galvez R, Colcombe S, Swain RA, Kramer AF, Greenough WT. Exercise, experience and the aging brain. Neurobiol Aging. 2002 Sep-Oct;23(5):941-55.

\section{Title of table and figure}

Table 1. Summary of results and characteristics of the included studies

Figure 1. Flow diagram of the included studies. 